\title{
Response selection in choice reaction time: Test of a buffer model
}

\author{
STEVEN P. MEWALDT \\ Marshall University, Huntington, West Virginia 25701 \\ and \\ CHERYL L. CONNELLY and J. RICHARD SIMON \\ University of Iowa, Iowa City, Iowa 52240
}

\begin{abstract}
Two experiments were conducted to test a buffer model of response selection. Subjects reacted to the onset of one of six possible visual stimuli by pressing either a left- or a righthand key. Two stimuli were assigned to one key (two-item set) and four stimuli were assigned to the other key (four-item set). An irrelevant monaural tone accompanied the visual stimulus in Experiment 1 but not in Experiment 2. Results of Experiment 1 showed that reactions were faster when the location of the tone corresponded with the response than when it did not, and the difference between these corresponding and noncorresponding conditions was greater for the two-item set than for the four-item set. Results supported the notion that the response selection process involved a serial self-terminating search of response buffers and that the tone determined the buffer searched first. In both experiments, reactions were faster to stimuli from the twoitem set than to stimuli from the four-item set.
\end{abstract}

The time period between the presentation of a stimulus and the initiation of a response in a choice reaction time (RT) task is commonly viewed as being filled with a series of independent stages or cognitive processes. For example, Stemberg (1969) has suggested that choice RT is composed of preprocessing or encod. ing, comparison or stimulus identification, response selection, and response execution. While numerous models have been proposed for these processing stages, most of the attention has been devoted to stimulus identification (Smith, 1968). The present paper is aimed at elaborating and testing a model of response selection originally proposed by Simon, Acosta, Mewaldt, and Speidel (1976).

The main experiment employs an irrelevant directional cue presented simultaneously with a relevant symbolic cue in order to bias the response selection process. There is considerable empirical evidence that suggests that subjects tend to respond initially to the directional component of a stimulus rather than to its symbolic content (Simon, 1970; Simon \& Craft, 1970).

A pilot study for this article was submitted by C. L. Connelly as an honors thesis under the direction of J. R. Simon at the University of Iowa. The authors wish to thank Enrique Acosta, Jr., and Julie Ferguson for their assistance in data collection. We also wish to thank an anonymous reviewer for comments on an earlier draft of this paper. This article is an extension of a paper presented at the annual meeting of the Midwestern Psychological Association, Chicago, May 1979. Requests for reprints should be sent to Steven P. Mewaldt, Department of Psychology, Marshall University, Huntington, West Virginia 25701.
For example, responses to a symbolic stimulus commanding a left- or right-hand response are faster when the location of the stimulus corresponds with the response than when it does not (Simon \& Rudell, 1967). The effect of this irrelevant directional cue seems to be confined to the response selection stage of information processing (Acosta \& Simon, 1976; Simon, Acosta, Mewaldt, \& Speidel, 1976; Simon \& Pouraghabagher, 1978). We propose, therefore, that the directional cue be used as a tool to investigate the response selection process.

Essentially, our model employs a scanning notion as a means of describing the response selection process. It proposes that a response "buffer" is established for each acceptable response in a choice RT task. Therefore, in a task that requires pressing either a left- or right-hand key, two buffers are created, one for the left- and one for the right-hand response. Each buffer contains a representational listing of the stimuli associated with that particular response. For example, if the stimulus " $\mathrm{X}$ " indicates a left-key response and the simulus " $\mathrm{O}$ " indicates a right-key response, a representation of " $\mathrm{X}$ " is contained within the left buffer and a representation of " $O$ " is contained within the right buffer. It is assumed that once a presented stimulus has been identified, each of the response buffers is searched with a serial, selfterminating scan until the matching representation is located and the associated response is selected. It is further assumed that an irrelevant directional cue associated with a stimulus affects RT by biasing the subject to search first the buffer that corresponds to the 
irrelevant cue. Thus, when the relevant and irrelevant cues correspond, RT will be relatively fast because the correct buffer will be searched first. However, when the relevant and irrelevant cues do not correspond, RT will be relatively slow, since the incorrect buffer will be the first searched.

The present research was designed as a test of this buffer model of response selection. The test involved assigning unequal numbers of stimuli to key press responses by the left or right hand. Specifically, two visual stimuli were assigned to a response by one hand (henceforth called the two-item set) and four visual stimuli were assigned to a response by the other hand (the four-item set). Each time one of these stimuli was presented, it was accompanied by a monaural tone that provided the irrelevant directional cue. Sometimes the tone was presented to the ear corresponding to the response to be made on that trial (henceforth called corresponding trials), and sometimes the tone was presented to the ear that did not correspond to the response (noncorresponding trials). The model makes predictions concerning the relationships among the RTs observed for the four different combinations of relevant and irrelevant information. These predictions are based on the assumptions that the locus of the tone determines the buffer searched first and that RT is a direct function of the number of items scanned during response selection.

Table 1 shows the presumed average number of items that must be scanned in order to identify a stimulus from a two- or four-item set under conditions in which the irrelevant directional cue corresponds with the response and conditions in which it does not. For example, consider the case in which a stimulus from a two-item set is accompanied by a noncorresponding tone. The tone biases the subject to search first through the four-item buffer, which, in this instance, is the incorrect buffer. We assume that all four items in this buffer are scanned before the two-item buffer is searched. The stimulus is finally located as either the first or second item in the two-item buffer. In this example, then, the subject has to scan an average of 5.5 items $(4+1.5)$ before the correct response can be selected and executed. If a stimulus from a two-item set is accompanied by a corresponding tone, the correct

Table 1

Predicted Average Number of Items Scanned to Locate a Stimulus From a Two- or Four-Item Set

\begin{tabular}{lcc}
\hline $\begin{array}{c}\text { Correspondence of } \\
\text { Tone and Response }\end{array}$ & $\begin{array}{c}\text { Two-Item } \\
\text { Set }\end{array}$ & $\begin{array}{c}\text { Four-Item } \\
\text { Set }\end{array}$ \\
\hline Noncorresponding (N) & 5.5 & 4.5 \\
Corresponding (C) & 1.5 & 2.5 \\
N - C Difference & 4.0 & 2.0 \\
\hline
\end{tabular}

Note-Model assumes that the location of the irrelevant tone determines the buffer searched first and that the search is selfterminating. buffer (i.e., the two-item buffer) is searched first, and the stimulus is located after scanning an average of 1.5 items. The average number of items that must be scanned to locate a stimulus from the four-item set is calculated in the same manner, and these figures are presented in the right column of Table 1.

Thus, a major prediction that derives from our model of response selection is that the difference in RT between corresponding and noncorresponding trials should be greater for stimuli from the two-item set than for stimuli from the four-item set. This prediction is based on two assumptions: (1) The location of the irrelevant tone determines the buffer searched first, and (2) RT is a function of the number of items scanned. As Table 1 indicates, the difference in items scanned between corresponding and noncorresponding trials should be greater for stimuli from the two-item set than for stimuli from the four-item set.

\section{EXPERIMENT 1}

\section{Method}

Subjects. The subjects were 16 male and 16 female righthanded students enrolled in introductory psychology at the University of Iowa, who participated to fulfill a course requirement. All students reported having normal or corrected-to-normal vision and hearing.

Apparatus. The apparatus provided a measure of choice RT in a task that required the subject to press either a left-or righthand key in response to the onset of one of six visual stimuli. The stimuli $(?,=,:, *, 8$, and +$)$ were presented by means of a Monsanto MDA-111 alphanumeric readout that was installed in an aluminum panel at eye level and approximately $50 \mathrm{~cm}$ from the subject. The stimuli had a character height of $8.9 \mathrm{~mm}$ and a stroke width of $1.0 \mathrm{~mm}$. A warning signal was provided by a green light installed $5 \mathrm{~cm}$ directly above the readout. Simultaneously with the visual stimuli, a $625-\mathrm{Hz}$ tone at $100 \mathrm{~dB}$ (re .0002 microbar) was presented to the subject's left or right ear. The tone was generated by a Hewlett-Packard audio oscillator (Model $200 \mathrm{AB}$ ) and was presented through Grason Stadler TDH-39 10Z earphones. Two microswitch keys were positioned on a table directly in front of and $9 \mathrm{~cm}$ on either side of the readout. Subjects nperated the keys with their left and right index fingers.

Stimuli were read from punched paper tape by a Digitronics Model 2060 perforated tape reader. Paper tape was also used to recorded responses and RTs (in milliseconds) via a Roytron Model 518 tape punch. Control of stimulus presentation and data acquisition was provided by BRS-Foringer solid state logic (Digibits 200 series).

Procedure. Subjects were seated in a soundproof booth at a table facing the display panel. They were told that one of six stimuli would appear on the screen and that, simultaneously, a tone would be presented to the left or right ear. They were instructed to respond to the visual stimulus as rapidly and accurately as possible. Four of the stimuli were assigned to one response key (the four-item set), and two stimuli were assigned to the other response key (the two-item set). To aid the subject in remembering the stimulus-response assignments, each set of stimuli was written on a $5 \times 8$ in. index card. These cards were taped to the display panel above the appropriate response key during the familiarization and practice trials, but they were removed during the test trials.

Subjects were first given 12 familiarization trials in which the six stimuli were each presented twice in a random order, once 
accompanied by a tone to the left ear and once accompanied by a tone to the right ear. This was followed by a 32-trial practice block in which each stimulus of the two-item set was presented eight times and each stimulus of the four-item set was presented four times, thereby requiring an equal number of left- and rightkey responses. Each stimulus was accompanied by an equal number of left- and right-ear tones. Finally, subjects were given 16 warm-up trials ( 8 left- and 8 right-key responses), which were followed immediately by 160 experimental trials. The experimental trials consisted of five blocks, each containing 32 trials. Each block contained the same number of presentations of each stimulus as did the practice block, but the stimuli were presented in a different random order in each block.

All trials began with a $150-\mathrm{msec}$ warning light, followed $1 \mathrm{sec}$ later by the simultaneous presentation of the visual stimulus and the monaural tone. These stimuli were both terminated by the subject's response. Two seconds after the response, the warning light for the next trial was presented.

The experimental design consisted of two within-subjects factors and two between-subjects factors. The within-subjects factors were ear stimulated (left or right) and set size (either the two- or the four-item set assigned to a particular response key). The between-subjects factors were response assignment and set content. For half the subjects, the two-item set was assigned to a left-hand response and the four-item set was assigned to a right-hand response. The other half of the subjects received the reverse assignment. To balance possible differences between particular stimuli, four different two- and four-item sets were used. The particular assignment of stimuli to the two- and fouritem sets was determined at random with the restrictions that each stimulus was in the two-item set at least once, and no pairing was repeated. Two males and two females were then assigned at random to each of the eight combinations of the two response assignments and the four set-content conditions.

\section{Results and Discussion}

Median RTs were computed for each subject for each of the four combinations of set size and tone-response location correspondence. Trials on which errors were made (4\%) were excluded from these computations. The error rates for the separate treatment combinations were virtually identical.

Table 2 presents the data collapsed across responding hand so that the right- and left-hand responses are represented equally in all of the means. An analysis of variance indicated a significant main effect of correspondence $[F(1,30)=69.37, \mathrm{p}<.001, \mathrm{MSe}=326.60]$; that is, responses were faster when the tone was presented to the side corresponding to the correct response than when it was presented to the noncorresponding side ( 524 vs. $551 \mathrm{msec}$ ). Of greatest interest, however, is the significant Set Size by Correspondence interaction $[\mathrm{F}(1,30)=16.10, \mathrm{p}<.001, \mathrm{MSe}=160.46] ;$ that is, the difference in RT between corresponding and noncorresponding trials for the two-item set was significantly larger than for the four-item set ( 36 vs. $17 \mathrm{msec}$ ). This finding confirms one of the major predictions derived from our buffer model of response selection.

The data, however, do not fit our model precisely. As pointed out previously, the model assumes that the RT for a particular treatment is determined by the average number of items scanned during response selection; that is, the slowest RT should be associated
Table 2

Reaction Time (in Milliseconds) as a Function of Correspondence of Tone-Response Relationship and Size of Stimulus Set: Experiment 1

\begin{tabular}{|c|c|c|c|}
\hline $\begin{array}{l}\text { Correspondence of } \\
\text { Tone and Response }\end{array}$ & $\begin{array}{l}\text { Two-Item } \\
\text { Set }\end{array}$ & $\begin{array}{l}\text { Four-Item } \\
\text { Set }\end{array}$ & Mean \\
\hline $\begin{array}{l}\text { Noncorresponding }(\mathrm{N}) \\
\text { Corresponding }(\mathrm{C})\end{array}$ & $\begin{array}{l}540 \\
504\end{array}$ & $\begin{array}{l}561 \\
544\end{array}$ & $\begin{array}{l}551 \\
524\end{array}$ \\
\hline Mean & 522 & 553 & \\
\hline$N-C$ Difference & 36 & 17 & \\
\hline
\end{tabular}

with the treatment that involved scanning the largest number of items. Table 1 shows the average number of items presumed to be scanned for each of the four combinations of set size and tone-response location correspondence. Note that identifying a stimulus from a two-item set when the tone location is noncorresponding requires scanning the greatest number of items (5.5) and should, therefore, produce the slowest RT. In contrast, identifying a stimulus from a two-item set when the stimulus is accompanied by a corresponding tone involves scanning the fewest number of items (1.5) and should, presumably, produce the fastest RT. Similarly, identifying a stimulus from a four-item set under corresponding and noncorresponding tone conditions involves scanning 2.5 and 4.5 items, respectively, and should, therefore, produce RTs of intermediate magnitude. The reader will note that a rank ordering of the RTs observed for these treatment conditions (Table 2) does not agree with the predictions based on the average number of items scanned. Obviously, some other factor, not included in our model, affected RT. The analysis of variance identified this factor as being associated with set size. Table 2 shows that subjects responded significantly faster to stimuli from the two-item set than to stimuli from the four-item set $(522$ vs. $553 \mathrm{msec})$ $[\mathrm{F}(1,30)=37.45, \mathrm{p}<.001, \mathrm{MSe}=784.34]$.

In retrospect, we might have expected that this setsize effect would occur for two reasons. First, subjects might tend to adopt a strategy of initially scanning the set with the fewer number of alternatives (i.e., the twoitem set). This bias could exist independently of the biasing effect of the irrelevant tone. Second, it is possible that a set-size effect might be produced by differences in stimulus probability. Because we wanted to equate the frequency of left- and right-key responses, the stimuli in the two-item set were presented twice as frequently as the stimuli in the four-item set. The greater frequency (i.e., probability) of stimuli in the two-item set could account for the faster RT (Smith, 1968), although there is evidence that stimulus probability effects do not completely account for set-size effects (Biederman \& Stacy, 1974). Several studies suggest that the effects of stimulus probability manipulations are isolated in either the stimulus encoding or the stimulus identification stage of processing (e.g., Miller \& Pachella, 
1973; Theios, Smith, Haviland, Traupmann, \& Moy, 1973; Theios \& Walter, 1974; Acosta \& Hinrichs, Note 1). Our model, of course, was concerned only with the response selection stage and did not attempt to account for effects that may have occurred during an earlier stage of processing. Therefore, in order to confirm the magnitude of the set-size effect observed in Experiment 1, we decided to conduct a control experiment in which no tone was present to bias response selection. If the setsize effect were replicated, we could then adjust the data from Experiment 1 accordingly.

\section{EXPERIMENT 2}

Experiment 2 was essentially a replication of Experiment 1 , except that the tones were not presented with the visual stimuli. The experiment was designed to provide an estimate of the magnitude of the set-size effect in a context that was free from any response bias produced by the irrelevant tone.

\section{Method}

The materials and procedure were identical to those used in Experiment 1. Subjects wore the headset, as in Experiment 1; however, it was disconnected. The subjects were 16 students at the University of Iowa who participated in order to fulfill a course requirement.

\section{Results and Discussion}

Because the same stimulus tapes were used in both experiments, it was possible to score the data from Experiment 2 in the same manner as the data in Experiment 1 were scored. That is, trials in which the tone and response corresponded in Experiment 1 were scored as if they were corresponding trials in Experiment 2. Similarly, trials that were noncorresponding in Experiment 1 were scored as if they were noncorresponding in Experiment 2. The data are presented in Table 3. An analysis of variance indicated that set size was the only significant effect $[F(1,14)=12.73, p<.01$, MSe $=1,310.26]$. RTs to stimuli in the two-item set were $32 \mathrm{msec}$ faster than those to stimuli in the fouritem set $(511$ vs. $543 \mathrm{msec})$. This difference agrees almost exactly with the $31-\mathrm{msec}$ difference observed in Experiment 1.

Since the magnitude of the set-size effect was so consistent in the two experiments, we must conclude that it was not influenced by the irrelevant directional

Table 3

Reaction Time (in Milliseconds) as a Function of Size of Stimulus Set With "Correspondence" as a Mock Variable: Experiment 2

\begin{tabular}{lccc}
\hline \multicolumn{1}{c}{ Two-I tem Four-Item } & \\
"Correspondence" & Set & Set & Mean \\
\hline "Noncorresponding" (N) & 513 & 543 & 528 \\
"Corresponding" (C) & 508 & 543 & 525 \\
Mean & 511 & 543 & \\
\hline
\end{tabular}

Table 4

Reaction Time (in Milliseconds) From Experiment 1 Adjusted to Remove the Set-Size Effect

\begin{tabular}{lccc} 
Correspondence of & \multicolumn{2}{c}{ Two-Item } & Four-Item \\
Tone and Response & Set & Set & Mean \\
\hline Noncorresponding (N) & 540 & 530 & 535 \\
Corresponding (C) & 504 & 513 & 508 \\
Mean & 522 & 522 & \\
N - C Difference & 36 & 17 & \\
\hline
\end{tabular}

Note-Adjustment involved subtracting $31 \mathrm{msec}$ from $R T S$ to the four-item set.

cue that affected response selection in Experiment 1. Indeed, our results suggest that the set-size effect is probably located in an earlier processing stage (i.e., stimulus encoding or stimulus identification). We propose, therefore, that subjects enter the response selection stage approximately $30 \mathrm{msec}$ sooner when they receive a stimulus from the two-item set than when they receive a stimulus from the four-item set. Subsequently, response selection proceeds as described in our model. Therefore, in order to compare response selection times for the two- and four-item sets in Experiment 1, it is necessary to remove differences that might be related to earlier processing stages. This can be accomplished by adjusting the mean RTs for the four-item set by our estimate of the set-size effect, that is, by subtracting $31 \mathrm{msec}$ from the RTs for the four-item set.

Table 4 displays the adjusted data. It can be seen that, now, not only is the difference in RT between corresponding and noncorresponding trials as predicted (of course, this is unchanged from before), but also the ordering of the means is exactly as predicted based on assumptions regarding the average number of items scanned under the four treatment conditions, as presented in Table 1. Furthermore, by comparing the predicted difference between any two conditions in number of items scanned (Table 1) with the observed difference in RT between the same two conditions (Table 4), one can obtain an estimate of the scanning rate per item. For example, there is a four-item difference in the number of items scanned between noncorresponding and corresponding tone locations in the two-item set. The RT difference between these two conditions is $36 \mathrm{msec}$. Dividing 36 by 4 , we obtain an average scanning rate of $9 \mathrm{msec} /$ item. Similar comparisons of any pair of conditions in Table 1 with the corresponding RT difference computed from Table 4 yields an almost identical estimate of the scanning rate. In other words, if one were to plot corrected RT as a function of predicted number of items scanned, one would find that a straight line with a slope of $9 \mathrm{msec}$ fits the data almost perfectly.

\section{GENERAL DISCUSSION}

This research was designed to test a buffer model of 
response selection proposed earlier (Simon, Acosta, Mewaldt, \& Speidel, 1976). The model was developed to account for a potent effect observed in previous RT studies, all of which employed a one-to-one stimulusresponse mapping. In these studies, subjects responded faster to a symbolic stimulus when its location (e.g., left or right side) corresponded with the required response (e.g., left or right key) than when it did not. According to the model, a response buffer is established for each alternative response. Each buffer contains a representation of the stimuli assigned to that response. After a stimulus has been presented and identified, subjects search the response buffers until they locate the stimulus in one of the buffers. They then execute the response associated with that buffer. The location of the stimulus provides an irrelevant directional cue that biases the subject to search either the left- or right-response buffer first. When the buffer that corresponds to the response is searched first, RT is relatively fast. However, when the noncorresponding buffer is searched first, RT is slowed, since an additional item must now be scanned before the stimulus is located.

To test this buffer model, we used a many-to-one stimulus-response mapping paradigm, with two stimuli assigned to one response and four stimuli assigned to another response. We reasoned that if the irrelevant directional cue determined the buffer searched first, then the consequences of searching an incorrect buffer should vary, depending on the number of items in that buffer. Specifically, we predicted that the differences in RT between corresponding and noncorresponding trials would be greater for stimuli from the two-item set than for stimuli from the four-item set. Our results confirmed this prediction and thus supported the buffer model.

Furthermore, after we corrected for the longer identification time for items from the four-item set, our data showed a nearly perfect relationship between the RTs for the various treatment conditions and the number of items presumably scanned in each condition. The scanning rate, calculated to be approximately $9 \mathrm{msec} / \mathrm{item}$, is considerably faster than the 17- to $40-\mathrm{msec}$ rates that other investigators have reported for stimulus identification scanning (Klatsky \& Smith, 1972; Sternberg, 1969; Theios et al., 1973). It may be that searching the response buffers is a faster process than stimulus identification scanning. Stimulus identification might be thought of as comparing an internal representation of some external stimulus with a fixed representation (i.e., a template) of that stimulus. If one assumes that these two representations of the stimulus are not identical, then one might expect a relatively slow comparison or scanning rate. Searching the response buffers, on the other hand, might be thought of as comparing two identical templates (the template used in stimulus identification with the template contained in the response buffer), and thus, the determination of a match could be easier and faster. It should also be noted that the estimated scanning rate was based on the observed difference between corresponding and noncorresponding trials. We know from previous studies that the size of this difference varies depending on factors such as task complexity, S-R compatibility, and strength of the directional cue (Simon, 1970; Simon, Acosta, Mewaldt, \& Speidel, 1976; Simon, Mewaldt, Acosta, \& $\mathrm{Hu}, 1976)$. The important finding, then, is not our estimate of the scanning rate per se. Rather, the point is that the data fit a model that assumes a serial selfterminating search through response buffers ${ }^{1}$ for which a directional cue determines the buffer searched first. Alternative models (e.g., random selection of a response buffer, exhaustive search, searching the smaller buffer only, etc.) do not fit the present data.

One final word: we assume that, in Experiment 2, the buffer searched first on a given trial was chosen at random, since no irrelevant tone was present to bias the buffer selection. Consequently, each treatment condition should contain a mixture of correct and incorrect buffer selections. One might expect, therefore, that the RTs observed in Experiment 2 should fall somewhere between the corresponding and noncorresponding RTs in Experiment 1. This logic, however, does not necessarily follow. First, the different groups involved in the two experiments were not necessarily comparable in average RT. Second, and most important, is the evidence from previous research that the relation of no-cue RT to corresponding and noncorresponding RT depends upon whether these three treatment combinations are included within the same trial block or whether the no-cue treatment is run as a separate block. In experiments in which the three kinds of trials have all been mixed within the same block, the RT for the no-cue treatment has been intermediate between the corresponding and noncorresponding RTs, just as our buffer model would predict (Simon \& Craft, 1970; Simon \& Pouraghabagher, 1978; Wallace, 1971). ${ }^{2}$ On the other hand, in experiments in which the no-cue treatment has been run in a separate trial block, the RTs have tended to be more comparable to the corresponding trials, which is the pattern observed in the present study (Craft \& Simon, 1970; Simon \& Small, 1969). Simon and Craft (1970) have suggested that the relation of no-cue RT to corresponding and noncorresponding RT may be a function of the amount of uncertainty associated with the no-cue trials (Garner, 1962). That is, when the no-cue trials are run in a separate block, there is uncertainty only with regard to the relevant stimulus; thus RT may be faster than in a mixed block in which there is uncertainty with regard to both the relevant and irrelevant stimulus.

\section{REFERENCE NOTE}

1. Acosta, E., Jr., \& Hinrichs, J. V. Probability effects in choice reaction time: Stimulus, response, or $S-R$ association frequency? Paper presented at the meeting of the Midwestern Psychological Association, Chicago, May 1977. 


\section{REFERENCES}

Acosta, E., JR., \& Simon, J. R. The effect of irrelevant information on the stages of processing. Journal of Motor Behavior, 1976, 8, 181-187.

Biederman, I., \& Stacy, E. W., Jr. Stimulus probability and stimulus set size in memory scanning. Journal of Experimental Psychology, 1974, 102, 1100-1107.

Craft, J. L., \& Simon, J. R. Processing symbolic information from a visual display: Interference from an irrelevant directional cue. Journal of Experimental Psychology, 1970, 83, 415-420.

GARNER, W. R. Uncertainty and structure as psychological concepts. New York: Wiley, 1962.

Klatzky, R. L., \& Smith, E. E. Stimulus expectancy and retrieval from short-term memory. Journal of Experimental Psychology, 1972, 94, 101-107.

Miller, J. O., \& Pachella, R. G. Locus of the stimulus probability effect. Journal of Experimental Psychology, 1973, 101, 227-231.

Simon, J. R. Stereotypic reactions in information processing. In L. E. Smith (Ed.), Psychology of motor learning. Chicago: The Athletic Institute, 1970.

Simon, J. R., Acosta, E., JR., Mewaldt, S. P., \& Speidel, C. R. The effect of an irrelevant directional cue on choice reaction time: Duration of the phenomenon and its relation to stages of processing. Perception \& Psychophysics, 1976, 19, 16-22.

Simon, J. R., \& Craft, J. L. Effects of an irrelevant auditory stimulus on visual choice reaction time. Journal of Experimental Psychology, 1970, 86, 272-274.

Simon, J. R., Mewaldt, S. P., Acosta, E., JR., \& Hu, J. M. Processing auditory information: Interaction of two population stereotypes. Journal of Applied Psychology, 1976, 61, 354-358.

Simon, J. R., \& Pouraghabagher, A. R. The effect of aging on the stages of processing in a choice reaction time task. Journal of Gerontology, 1978, 33, 553-561.
Simon, J. R., \& Rudell, A. P. Auditory S-R compatibility: The effect of an irrelevant cue on information processing. Journal of Applied Psychology, 1967, 51, 300-304.

Simon, J. R., \& Small, A. M., JR. Processing auditory information: Interference from an irrelevant cue. Journal of Applied Psychology, 1969, 53, 433-435.

Smith, E. E. Choice reaction time: An analysis of the major theoretical positions. Psychological Bulletin, 1968, 69, 77-110.

Sternberg, S. Memory scanning: Mental processes revealed by reaction-time experiments, American Scientist, 1969, 57, 421-457.

Theios, J., Smith, P. G., Haviland, S. E., Traupmann, J., \& MoY, M. C. Memory scanning as a serial self-terminating process. Journal of Experimental Psychology, 1973, 97, 323-336.

Theios, J., \& Walter, D. G. Stimulus and response frequency and sequential effects in memory scanning reaction times. Journal of Experimental Psychology, 1974, 102, 1092-1099.

Wallace, R. J. S-R compatibility and the idea of a response code. Journal of Experimental Psychology, 1971, 88, 354-360.

\section{NOTES}

1. It is possible that the procedure followed during the familiarization and practice trials (i.e., displaying each set of stimuli above the appropriate response key) may have biased subjects toward following this particular search pattern.

2. In fact, we included a within-block no-cue condition in a pilot experiment that had essentially the same design as the present experiment. As predicted, the no-cue condition produced in termediate RTs.

(Received for publication November 14, 1979; revision accepted May 23, 1980.) 